\title{
Phase calibration of the EISCAT Svalbard Radar interferometer using optical satellite signatures
}

\author{
J. M. Sullivan ${ }^{1}$, N. Ivchenko ${ }^{2}$, M. Lockwood ${ }^{3}$, T. Grydeland ${ }^{4}$, E. M. Blixt ${ }^{4}$, and B. S. Lanchester ${ }^{1}$ \\ ${ }^{1}$ School of Physics and Astronomy, University of Southampton, Southampton, Hampshire, UK \\ ${ }^{2}$ Alfvén Laboratory KTH, Stockholm, Sweden \\ ${ }^{3}$ Rutherford Appleton Laboratory, Chilton, Didcot, Oxfordshire, UK \\ ${ }^{4}$ Dept. of Physics and Technology, University of Troms $\varnothing$, N-9037 Troms $\varnothing$, Norway \\ Received: 13 February 2006 - Revised: 27 June 2006 - Accepted: 4 July 2006 - Published: 20 September 2006 \\ Part of Special Issue "Twelfth EISCAT International Workshop"
}

\begin{abstract}
The link between natural ion-line enhancements in radar spectra and auroral activity has been the subject of recent studies but conclusions have been limited by the spatial and temporal resolution previously available. The next challenge is to use shorter sub-second integration times in combination with interferometric programmes to resolve spatial structure within the main radar beam, and so relate enhanced filaments to individual auroral rays.

This paper presents initial studies of a technique, using optical and spectral satellite signatures, to calibrate the received phase of a signal with the position of the scattering source along the interferometric baseline of the EISCAT Svalbard Radar. It is shown that a consistent relationship can be found only if the satellite passage through the phase fringes is adjusted from the passage predicted by optical tracking. This required adjustment is interpreted as being due to the vector between the theoretical focusing points of the two antennae, i.e. the true radar baseline, differing from the baseline obtained by survey between the antenna foot points. A method to obtain a measurement of the true interferometric baseline using multiple satellite passes is outlined.
\end{abstract}

Keywords. Radio science (Interferometry; Instruments and techniques) - Ionosphere (Auroral ionosphere)

\section{Introduction}

Through observations of enhancements in radar echoes at the EISCAT Svalbard Radar (ESR), evidence has been found of plasma instabilities within small scale filamentary structures, on the order of $100 \mathrm{~m}$ across, present in the cusp ionosphere (Grydeland et al., 2003). After initial studies into a connection between corresponding signatures seen on the nightside and aurora (Collis et al., 1991; Rietveld et al., 1991;

Correspondence to: J. M. Sullivan

(jms@phys.soton.ac.uk)
Cabrit et al., 1996), recent combined radar and optical studies of these filamentary enhancements, termed "Naturally Enhanced Ion-Acoustic Lines" or NEIALs, have identified a link with the occurrence of thin ray structure of similar scale size in the cusp region (Grydeland et al., 2004; Blixt et al., 2005). Enhanced echoes have been observed frequently at the ESR (Buchert et al., 1999; Sedgemore-Schulthess et al., 1999) and a review summarising the theories and observations of NEIALs from various observatories has been published by Sedgemore-Schulthess and St.-Maurice (2001).

The temporal variability and fine structure of these events does, however, lead to problems with time and spatial resolution when investigating the link with auroral structures in further detail. Standard ESR coding schemes have a temporal resolution of the order of $10 \mathrm{~s}$, whereas ion-acoustic enhancements have been shown to vary on the sub-second time scale (Grydeland et al., 2003). At 300-km altitude, a region of common NEIAL enhancement at the ESR, the width of the $42-\mathrm{m}$ radar antenna beam is around $6.75 \mathrm{~km}$ (Grydeland et al., 2003), making 100-m wide filaments impossible to resolve individually.

NEIALs are observed to have a very strong backscattering effect, with scattering cross sections 4-5 orders of magnitude above thermal levels (Grydeland et al., 2004). The high signal-to-noise ratios of these signatures mean that shorter integration times can be used to record them. By sampling raw (voltage-level) data, time series with resolution limited only by the interpulse period (IPP) of the transmission code can be obtained for each radar antenna. For the experiment discussed here the limiting IPP resolution is $10 \mathrm{~ms}$, whereas 0.2 -s radar data has been shown to be sufficient to follow the temporal resolution of NEIALs (Grydeland et al., 2004). Increased spatial resolution can be achieved by the use of new interferometry techniques. These employ the voltage time series from the two antennae of the ESR, which are approximately $130 \mathrm{~m}$ apart. The challenge now is to get radar observations down to the spatial scale of these enhancements and

Published by Copernicus GmbH on behalf of the European Geosciences Union. 
compare their location within the radar beam with the positions of auroral ray structures, in order to investigate previously found correlations in further detail.

For the interferometric technique, raw (voltage-level) samples are recorded. The resulting difference between phase received at each of the antennae can then be computed for the total scattered signal received in each integration period. In an ideal system, this phase difference would occur solely due to the difference in path length from the source of scattering to each receive antenna, as described in Eq. 1 in Appendix A. This could then be used to derive the location of a coherent scattering structure along the radar baseline within the main beam.

However, as for all interferometric receiver systems, sources of additional phase contribute to the final phase value recorded by the ESR, as the raw signal is transferred between different components and changed in frequency by mixing with the output of various local oscillators. This unknown system phase offset depends on factors such as signal path length (e.g. cable lengths, component group delays) and it can also vary slowly with environmental changes (e.g. changes in temperature).

It has been seen in previous studies that satellites passing through the main ESR beam cause strong coherent echoes similar in strength to natural enhancements. Indeed, many early observations of NEIALs were originally discounted as being due to satellite crossings (Foster et al., 1988). This paper presents a possible technique to use passing satellites, seen both optically and in radar spectra, to calibrate the values of phase recorded with the position of the scattering structures along the baseline. The satellite traces in the imagers, both remote-site and co-located (with the ESR), enable the theoretical phase values during the crossing through the radar beam to be predicted and compared with the recorded values. The system phase offset, representing the effect of the ESR receiver hardware on the phase of the outputted signal, can therefore be found. The technique of tracing optical signatures to calibrate radar phase has been employed previously for MST radars using aircraft signals (Chen et al., 2002), but this is the first study of its applicability to satellite signals and the ESR.

\section{Experimental method}

The ESR is an incoherent scatter radar facility, consisting of one fixed field-aligned 42-m antenna and one steerable 32-m antenna, approximately $130 \mathrm{~m}$ apart, on the Norwegian island of Spitsbergen within the Arctic circle. The location of the radar at $78^{\circ} 9^{\prime} \mathrm{N}, 16^{\circ} 2^{\prime} \mathrm{E}$ means that it is often covered by the ionospheric dayside cusp region, as is the case for the experimental run discussed here. This paper looks at the feasibility of the phase calibration technique using satellite signals seen optically and by the ESR in the morning hours of 26 January 2003, where NEIALs are present in the radar data and structured rayed aurora are measured with optical instruments.

\subsection{ESR interferometry}

In the interferometric set up, both ESR antennas are aligned along the magnetic field line, with look direction $181.0^{\circ}$ in azimuth and $81.6^{\circ}$ in elevation. Throughout the radar run, transmission is through the 32-m antenna only, while both antennae are set to receive. The radar programme "LT1HL" used here is a long-pulse experiment using two long pulses per IPP. For the 2002-2003 campaign season, the ESR system in LH1HL mode recorded integrated lag profile matrices (LPMs) at 2-s resolution from each of the two antennae at various frequencies. In order to access raw (voltage-level) data, the $11.25 \mathrm{MHz}$ IF (intermediate frequency) signal was split off and sampled independently in a MIDAS-W type receiver system (Holt et al., 2000), assembled by the University of Troms $\varnothing$. The power received in each antenna and the interferometric data products of phase difference and coherence (normalized cross spectrum) can then be obtained at sub-second resolution, according to the procedures explained in detail in Grydeland et al. (2004).

The geometry of the two antennae leads to lines of equal phase difference (fringes) being set up perpendicular to the radar baseline, separated by approximately $0.25 \mathrm{deg}$. As the length of the radar baseline is negligible compared to the range to the satellite signals, the fringes are assumed to be parallel for the altitudes involved here and the look direction to the signal can be taken to be identical for each antenna.

Power density spectra recorded over 0.2-s integrations with a range resolution of $42 \mathrm{~km}$ from 232 to $1702 \mathrm{~km}$, were used to identify satellite signals passing through the radar beam. Satellites are observed as an increase in power received in one or two range gates for several data dumps. Phase values (used now and in all future references to mean the difference between the phases received at the 32-m and 42-m antennas) at the central range gate and frequency of an observed satellite, were recorded along with the associated coherence and intensities.

\subsection{Optical instrumentation}

Two optical imagers were running throughout this campaign season. The University of Troms $\varnothing$ digital imager, "Odin", was mounted at the ESR site, co-aligned with the radar beam. Odin, described fully in Blixt et al. (2005), is a white light imager with field of view $14.3^{\circ}$ by $10.9^{\circ}$, producing a standard PAL video signal at 25 frames per second, which is then converted to digital PAL DV format in real time.

A second white light, narrow view imager was available as part of the Spectrographic Imaging Facility (SIF), an instrument platform run jointly by the University of Southampton and University College London, located at the Auroral Station (Sigernes et al., 2002) about $7 \mathrm{~km}$ north of the ESR at 
$78^{\circ} 12^{\prime} \mathrm{N}, 15^{\circ} 50^{\prime} \mathrm{E}$. This imager, described in Lanchester et al. (2003), has a field of view of $16^{\circ}$ by $12^{\circ}$ and also provides data at a resolution of 25 frames per second. Both imagers were fitted with a short-wavelength cut-off filter at $650 \mathrm{~nm}$, eliminating the long-lived emissions from atomic oxygen.

The imager fields of view were located onto the plane of the sky using routines to fit positions of known stars from the Smithsonian Astrophysical Observatory star catalogue to their observed positions in each imager. Software was developed to trace the path of a satellite in the Odin field of view and convert this into the azimuth, elevation coordinate system. It was then possible to map satellite paths through the radar phase fringes and produce a series of theoretical phase values over time. In the first instance, a nominal baseline vector $N$ was used. This was taken to be horizontal with a length of $132.5 \mathrm{~m}$, as measured by 2-D survey between the footpoints of the two antennae. Suitable satellites for optical phase calibration are required to give a long-lived coherent signal, passing close to the centre of the radar beam and travelling at a low enough velocity that the aliasing effects of the phase variation at 0.2 -s resolution are manageable.

The 7-km separation between the two narrow imagers used here means that for periods where Odin is completely obscured by cloud, the SIF camera f-o-v can be relatively clear with satellite signals visible throughout the period of observation. Part of this study is to investigate the feasibility of using SIF to confirm indirectly the track of a satellite though the radar beam. This will maximise the usable satellite passes in times of localised cloud, such as occurred during the event described here.

\subsection{Timing synchronisation}

For the 2002-2003 campaign season, the Odin imager was fitted with a GPS time feed, whereas the SIF platform clock was running independently. In order to compare the use of both optical stations, the timing must be synchronised between them. This was done by tracking satellites moving in a direction almost perpendicular to the optical baseline, in both cameras. The optical coordinate system is defined by the angles $\alpha$ and $\phi$, where $\alpha$ is the angle between the optical baseline and the look direction to the satellite for each camera, and $\phi$ is the angle between the plane containing optical baseline and magnetic zenith and the plane containing optical baseline and the satellite. Since the series of $\phi$ coordinates for such a satellite pass should be identical seen from either camera, the time difference between matching $\phi$ coordinates was used to synchronise the two image sequences.

In order to be sure that the satellites seen to be present in the radar spectra corresponded to those seen optically, time-corrected images from the two separated sites were also used to calculate an approximate range and altitude change per second by triangulation, to compare with range and frequency of observed signals in the radar.

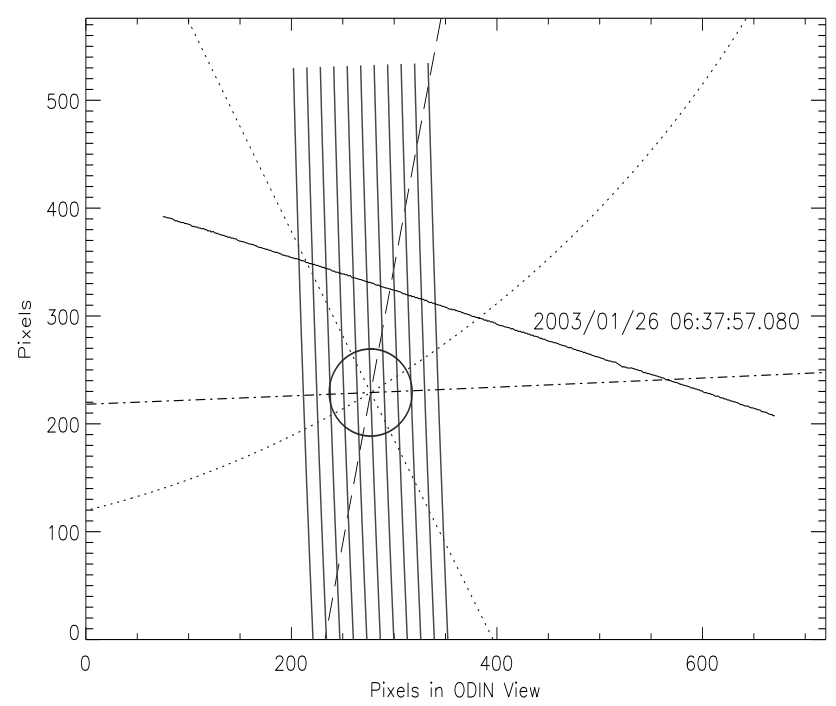

Fig. 1. Odin field of view; dash-dot line marks the cut of the sky by the plane containing the ESR interferometric baseline and the magnetic zenith direction, dashed line marks the cut of the sky by the plane containing the optical baseline and magnetic zenith direction, dotted line shows the meridian passing through the magnetic zenith, dotted arc shows the east-west line through the magnetic zenith, the solid circle of radius $0.8^{\circ}$ marks the half power level of the radar beam, solid thin lines mark the location of the ESR phase fringes and the darker black line shows the trace of the satellite from its position in each Odin frame

\section{Results and analysis}

For this particular event, Odin is obscured by cloud soon into the observation period, leading to only one suitable satellite being found for this analysis. This passed through the radar sidelobes with closest approach to the main beam at 06:38:07 UT. Although this means that full phase calibration is unlikely to be possible for the times of observed NEIALs, this satellite can be used to demonstrate the phase calibration technique. It is shown traced onto the Odin field of view (720 by 576 pixels) in Fig. 1.

The satellite was observed for $15.96 \mathrm{~s}$ in the Odin field of view, with an average angular velocity $0.774 \mathrm{deg} \mathrm{s}^{-1}$ and from the optical signature appears to be a large orbital object. Figure 2 shows the recorded coherence and power in the 32- $\mathrm{m}$ and 42-m antennae, recorded for the satellite signal in the radar data. It can be seen that high coherences and intensities in both antennae are recorded for a prolonged period of around $5 \mathrm{~s}$ as the satellite passes. The vertical dotted line is the time of maximum power as predicted from the satellite trace in the Odin optics, using a theoretical incoherent scatter antenna pattern. It can be seen that the radar signal matches the optical path of the satellite well, although there is an indication of an offset of about $0.25 \mathrm{~s}$ between the times of maximum power, mostly clearly seen in the $42-\mathrm{m}$ antenna. 

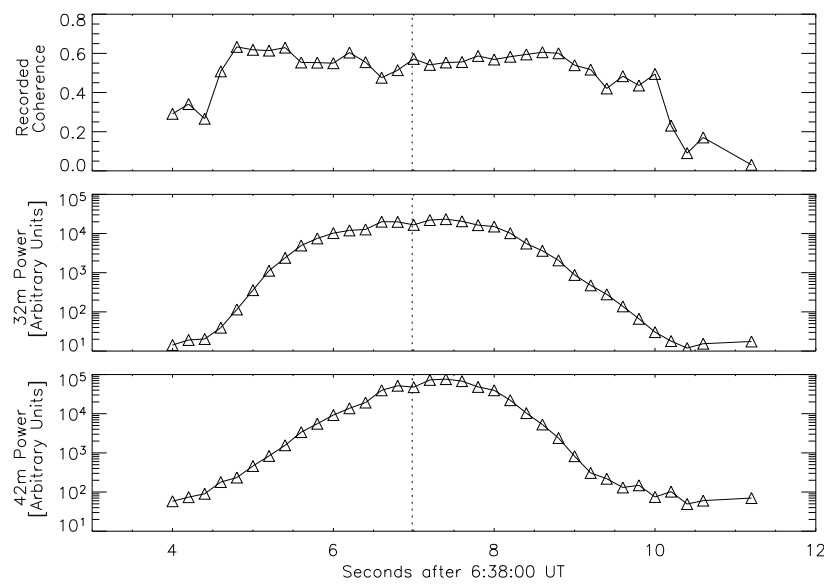

Fig. 2. Panels from top to bottom: (a) Observed coherence at central range and frequency of the satellite echo in ESR spectra, (b) Observed power of satellite echo in the ESR 32-m antenna, (c) Observed power of satellite echo in the ESR 42-m antenna.

One possible explanation is that the ESR and Odin GPS time were not exactly synchronised for this campaign season, which will make a large contribution to the phase offset between observed phase and optically predicted phase. The apparent time offset could also be related to the slight asymmetry present in the $42-\mathrm{m}$ beam power plot. This asymmetry could be due to slight modification of the antenna pattern by the position of the sub-reflector used to adjust the 42-m fieldaligned look-direction, or due to a build-up of snow inside the dish. A satellite rapidly changing in altitude as it crosses the ESR beam could also result in an asymmetric power plot similar to that observed in the bottom panel of Fig. 2, although from the constant range of the radar echoes, this was not the case for the satellite pass used here.

The top panel of Fig. 3 shows the recorded phase values from the ESR spectra (which vary between $-\pi$ and $+\pi$ ), overlaid onto the theoretical phase values. The theoretical phase values were predicted from the optical satellite coordinates using the theory outlined in Appendix A and a nominal horizontal ESR interferometric baseline as described in Sect. 2.2. It can be seen that even at 0.2-s resolution the satellite is passing through the phase fringes so quickly that only around 2 data points per phase loop are recorded. It should be noted here that for the frequency of the radar $(500 \mathrm{MHz})$, the wavelength is $60 \mathrm{~cm}$ and hence errors in the baseline of just a few centimeters will cause large errors in phase that will change as the angular coordinates of the satellite change.

The second panel of Fig. 3 shows the best match that can be obtained between the observed and predicted phase if the baseline is varied about its nominal value, which introduces a phase drift of $\delta_{0}$. In this case, the observed and predicted values follow each other well for the majority of the satellite pass, and then deviate at the edges where the recorded coherence and the power falls off.
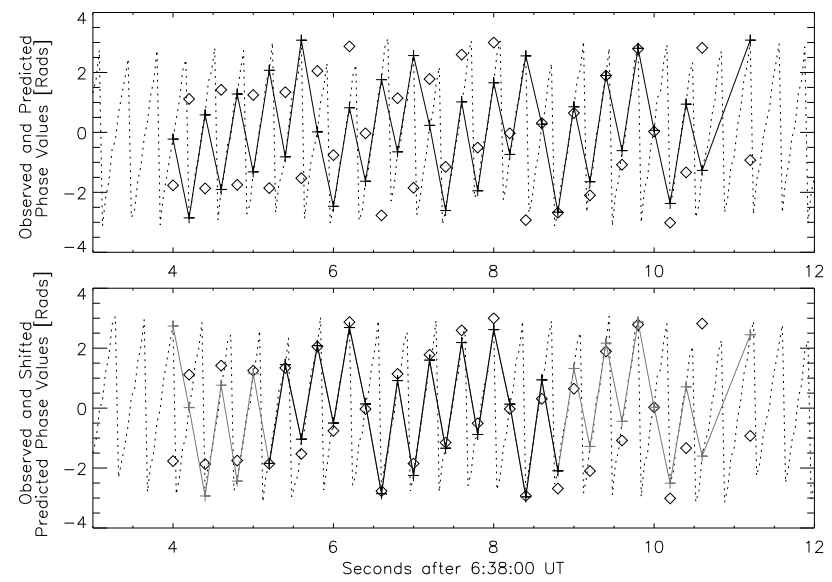

Fig. 3. Observed and optically predicted phase values. The diamonds mark the observed phase values taken directly from the ESR spectra. The dotted line shows the predicted phase values as taken from the optical satellite trace at 0.04-s resolution and the crosses, joined by the solid line, mark the interpolated phase value on this predicted trace, taken at the same time as the radar measurement. The first panel shows raw ESR and optically predicted phase values. The second panel shows the best match that can be obtained (by varying the baseline and the constant phase offset) between the central radar and optical points for which the power received by the 32-m antenna exceeded 1000 (arbitrary units, see Fig. 2), as shown in black. Data points with an associated power in the 32-m antenna below the chosen threshold of 1000 are joined by a grey line and are not taken into account in the fitting.

To obtain this best match, the optical predictions were corrected in two ways. First, the rate of change of phase was adjusted, thereby effectively varying the baseline, to give the lowest mean square difference between observed and predicted phase values. In reality the radar baseline is not perfectly horizontal, with the antennae being situated on uneven ground and having collecting feeds at different heights. To replicate exactly the observed rate of phase change, the baseline length used should be the distance between the actual focusing points of the antennae, which is not directly measurable by survey. Secondly, the optical predictions were shifted by a certain constant phase, which represents the best estimate of phase offset due to the ESR receiver system and timing synchronisation errors between ESR and Odin for this campaign. This was found again by minimising the mean square difference between observed and predicted phases.

The top panel in Fig. 4 shows the mean square difference between observed phase and predicted phase, against phase offset, once the predicted phase has been adjusted for the best rate of phase change found. A clear minimum in the mean square difference can be seen corresponding to an ESR system phase offset of just over 0.1 rads. The second panel of Fig. 4 shows a Chi-square null hypothesis test on this data, with $\Delta \phi$ representing the range of values within the $95 \%$ confidence level. This range can be treated as the uncertainty 


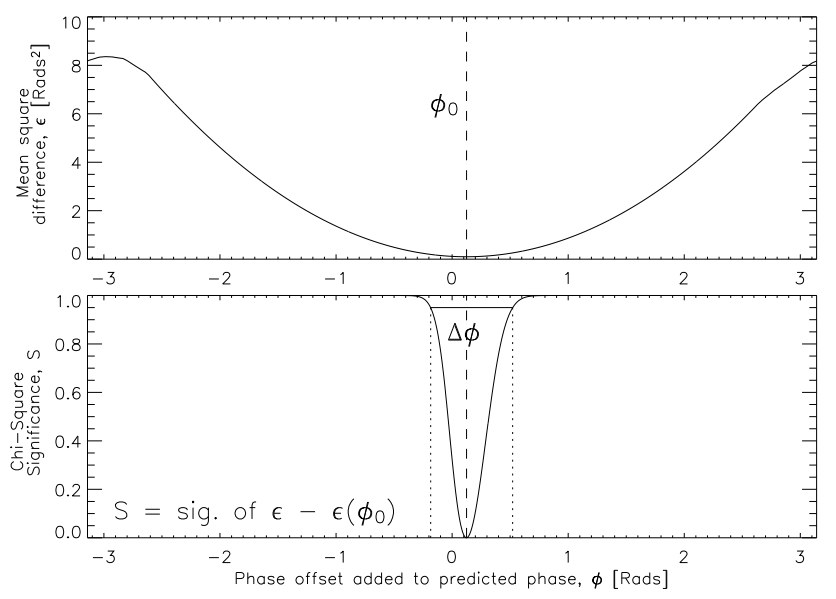

Fig. 4. Top panel: Plot of mean square difference between observed and optically-predicted phase values, as a function of phase offset added to the predicted phase. Bottom panel: Plot of significance of the phase offset relative to the best offset found, $\phi_{0}: \Delta \phi$ is the confidence interval in $\phi_{0}$ for the $95 \%$ level.

in the best phase offset found. It can be seen that for only one satellite pass the final error on the phase offset is very large at \pm 0.4 rads. To reduce this to a usable accuracy more satellite passes are needed that give extended periods of consistent recorded phase values (see Appendix A).

Figure 5 shows how the best rate of phase change, used to produce Fig. 4, was determined. Applied phase drift is here taken to mean the extra phase change added to the rate calculated using the nominal baseline. The effect of varying the applied phase drift is shown for the best phase offset found, the mean square difference between predicted and observed for all data points at best phase offset, and the width of the $95 \%$ confidence level for this offset. A drift of an extra $0.5 \mathrm{rads} \mathrm{s}^{-1}$, resulting in the lowest mean square difference at best phase offset, was used to produce the plots shown previously in Fig. 4. It can be seen that despite a clear minimum being evident, the best phase drift found with this limited number of data points during a single satellite pass, is not statistically significant at the $84 \%$ (1 sigma) confidence level.

Even with adjustment of the optically predicted data for the best phase drift and best phase offset, there is still some deviation from observations, with the minimum of the mean square difference curve never reaching zero, especially evident at the edges of the satellite pass. Figure 6 shows the square difference at best $\delta_{0}$ and $\phi_{0}$ for each phase data point against its corresponding antenna intensities and coherence value. It can be seen that at low intensities and low coherences below a certain threshold, the phase values recorded are not consistent with the best-fit predictions and are unreliable for use in phase calibration. Power recorded is shown to be a better measure of the quality of the data and its use for phase calibration than recorded coherence, which has a less

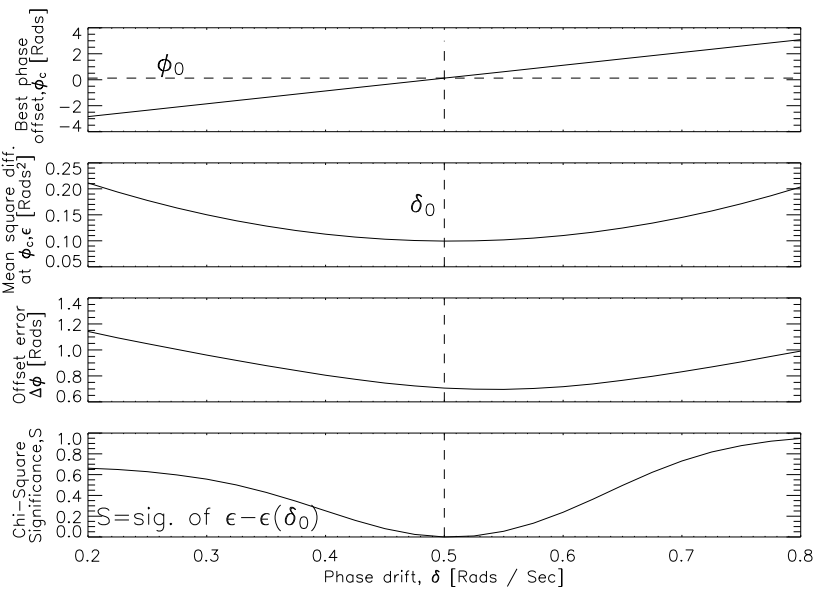

Fig. 5. From top to bottom : The effect of applied phase drift, $\delta$, to the optically predicted phase values on (a) The phase offset found (b) the mean square difference between observed phase and predicted phase with this offset (c) The width of the $95 \%$ confidence interval around the best phase offset found, and d) the Chi-square significance of the best applied phase drift found, $\delta_{0}$. Only phase values with an associated power in the 32-m antenna of over a chosen threshold of 1000 [Arbitrary units] were used for this analysis.
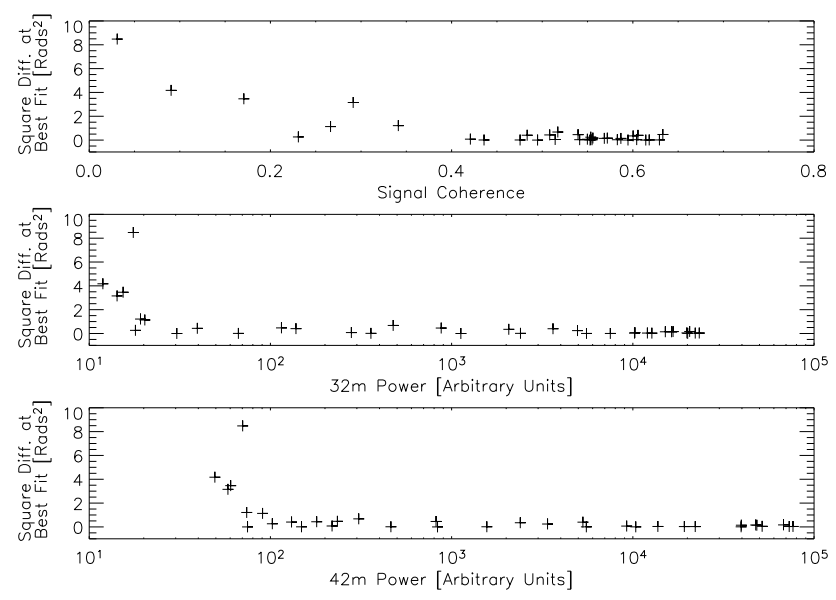

Fig. 6. Square difference between observed and best-match predicted phase value (i.e. adjusted for the best phase drift and phase offset) for each data point, as a function of (a) recorded coherence of that data point, (b) recorded power in the 32-m antenna for that data point and (c) associated recorded power in the 42-m antenna.

clearly defined threshold.

The consistent fit of radar phases to optical data relies on having high enough signal power in both the 32-m and 42-m dishes. If the signal level in either one of the receivers drops below a certain level relative to the noise, the resulting phase values will no longer be reliable. As shown in Fig. 2, the received power in the 42-m antenna is around 4 times that in the 32-m antenna, for the central section of the satellite pass. Hence, at the threshold level where the consistent fit starts to 


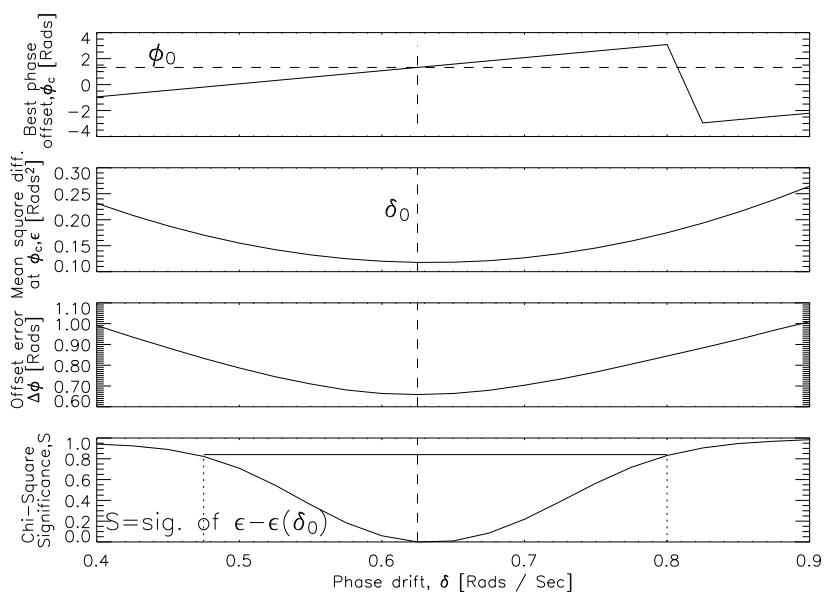

Fig. 7. Phase drift analysis as described in Fig. 5, but with a lower cut-off threshold power in the 32-m antenna of 100 [Arbitrary units]

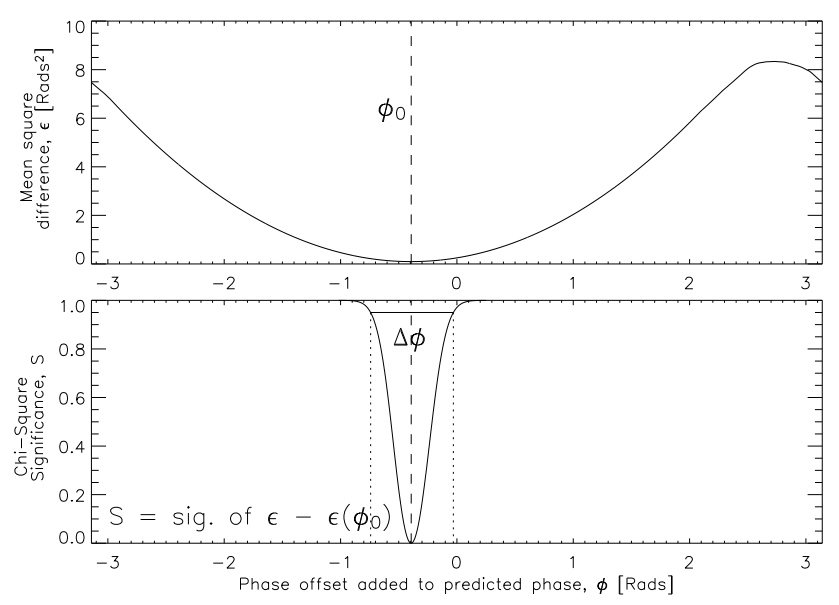

Fig. 8. Phase offset analysis as described in Fig. 4 but using the satellite position as traced from the remote camera on the SIF platform.

fail, we would expect the 42-m power to be roughly 4 times the 32-m power. This is consistent with the results shown in the bottom two panels of Fig. 6. In order to obtain consistent phase values, a good estimate for the threshold power required in the 32-m antenna is seen to be 100 [Arbitrary units], i.e. lower than the more restrictive power threshold of 1000 [Arbitrary units] that was used earlier in determination of the calibration values.

Figure 7 shows the same analysis as shown in Fig. 5, but with the lower power threshold employed to allow the use of more data points. It can be seen that this increased number of samples results in a statistically significant best phase drift at the $84 \%$ ( 1 sigma) level. Allowing the use of more data points at lower intensities results in a slightly different best drift rate, but both values lie within the $84 \%$ interval shown.
Figure 8 shows the corresponding analysis for the satellite signal traced in the SIF imager. This is used to assess the feasibility of using a remote site camera for satellite tracking if a radar co-aligned imager is unavailable or obscured by localized cloud. The drift required for the best match was found to be the same $\delta_{0}=0.5 \mathrm{rads} \mathrm{s}^{-1}$ as was found using the trace of the satellite directly in Odin, with the original threshold 32-m power of 1000 [Arbitrary units]. This corroborates the interpretation that this drift is due to errors in the defined radar baseline and not errors in the optics. It can be seen that the best phase offset found when using the drift adjusted SIF trace is 0.5 rads lower than the corresponding analysis. Taking into account the velocity at which the satellite is seen to cross the phase fringes in the radar, this corresponds to a timing error of $0.03 \mathrm{~s}$ between SIF and Odin. As the bi-static satellite tracking technique used to synchronise the two optical stations timing has an associated error of $\pm 0.02 \mathrm{~s}$, this is consistent within the bounds of error.

\section{Conclusions}

This paper presents an interferometry phase calibration technique, used for the first time at the ESR, and shows some initial results of its application. We have shown that it is possible to recreate observed phase values of a satellite passing through the ESR main dual-beam, using phase values predicted from optical satellite traces. In order to achieve a series of predicted phase values following recorded observations to the highest degree, it is necessary to apply:

[1] a phase offset representing phase added due to components in the ESR receiver system and due to timing synchronisation errors between ESR and Odin

[2] an added rate of phase change, arising due to inaccuracies in how the interferometric baseline is measured.

This paper has demonstrated a technique, using the results obtained for one satellite pass, to quantify these effects on the recorded phase values produced by the ESR. In order to calibrate recorded phase values with the position of coherent signals along the baseline, many satellite passes exhibiting high received coherences and power at the radar for several seconds will be needed, in order to obtain phase offset and drift values to usable accuracies.

It has also been shown that it is possible to use optical satellite traces from a remote-site camera to perform phase calibration, if the ESR co-aligned imager is obscured by localised cloud, to within the accuracy of the current technique. Images from the narrow view imager located at the Auroral Station as part of the Spectrographic Imaging Facility, can therefore be used to extend the database of satellites available for this technique in future campaigns.

For the satellite pass observed here, using the higher power cut-off, a phase offset of 0.1 rads to within a statistical accuracy of 0.4 rads has been shown, corresponding to an error in horizontal distance of approximately $400 \mathrm{~m}$ at $300-\mathrm{km}$ 
altitude. With, for example, a collection of $n=16$ such satellite passes with similar number of consistent recorded phase values, future work should allow this error to be reduced by $n^{\frac{1}{2}}$, i.e. to within $0.1 \mathrm{rads}$ and to within $100 \mathrm{~m}$ horizontally at 300-km altitude.

In using radar signal phases, it is important to consider whether ionospheric scintillation may have a significant effect on the observed phase of a satellite echo, and hence introduce a source of error into the calibration procedure described here. The interferometry technique is only sensitive to differences in phase at the two antennae, so to have a significant effect would require ionospheric density irregularities, as described in Tereshchenko et al. (1998), with a fieldperpendicular wavelength less than the beam separation at the height of the peak effect on the phase path. For a simple order of magnitude calculation we assume this to be at the Fregion peak, at an altitude of $300 \mathrm{~km}$. From similar triangles, and for the height of the satellite detected here (determined from the range of the echoes to be $500 \mathrm{~km}$ ), we find that irregularities of wavelength less than $d(500-300) / 500 \approx 50 \mathrm{~m}$ are needed to induce a significant phase path difference to the two antenna (where the antenna separation $d \approx 130 \mathrm{~m}$ ). If we assume that the irregularities are moving with a typical F-region convection speed of $1 \mathrm{kms}^{-1}$, these will give phase path differences that vary with periods of $0.05 \mathrm{~s}$.

Each interferometric data point, integrated over $0.2 \mathrm{~s}$, therefore covers at least 4 such irregularity cycles. This is not enough to average out the variations and implies a possible source of phase error due to scintillation in each data point. However our method relies on minimising the difference for the time series as a whole, rather than fitting each observed phase value individually to its optically predicted counterpart. If we use the power threshold of 100 [Arbitrary units] in the 32-m dish, as employed for Fig. 7, we obtain a time series used for the fitting of approximately $5 \mathrm{~s}$ in length. This therefore covers more than 100 effective scintillation cycles. The resulting phase differences will average out and have negligible effect on the overall fit.

One possible method of quantifying the relative contributions of phase difference errors for each radar data point due to ionospheric scintillation and the interferometry phase measurement processing, would be to feed a calibration signal into the ESR system at the local oscillator stage. The resulting phase measurement errors would then be caused by the interferometry processing technique alone. Whilst the introduction of a known signal would be a means of calibrating one part of the phase processing system, i.e. from the local oscillator stage to the phase interferometric data product, the use of satellites described in the present paper allows us to perform an "end to end" calibration of the entire radar interferometry system. With the high frequency of satellite passes over Svalbard, this technique also provides a means of routinely calibrating the phase for any interferometric data run during clear skies, without the need for extra calibration radar time or equipment setup.

The ESR frequency of $500 \mathrm{MHz}$ means that a phase difference of $2 \pi$ corresponds to a wavelength of just $60 \mathrm{~cm}$, and knowledge of the baseline to within this accuracy is essential for reliable phase calibration. Work is now ongoing to apply this technique to a large set of satellite passes observed both in ESR interferometric data and in the Odin and SIF fields of view. This will enable us to see whether an effective phase calibration can be obtained, in order to build up a selfconsistent map of the phase fringes throughout an ESR interferometry run. Appendix A presents a proposed method to obtain the true interferometric baseline in three dimensions, using a number of satellite passes at a range of orientations.

\section{Appendix A}

\section{Method for accurate estimation of interferometer baseline}

The wavelength of the radar signal $\lambda$ is $0.6 \mathrm{~m}$ and thus to measure phase difference accurately requires the interferometer baseline, between the foci of the two antennae, to be known to resolutions smaller than this (in three dimensions). Rather than trying to make this measurement directly, we here describe a technique that allows us to use the satellite measurements. To make a first fit, we use a nominal baseline vector, $N$, which is not to the above accuracy (and is assumed to be horizontal, which is not valid because the antennae foci are at slightly different heights) and then correct to the true baseline $\boldsymbol{B}$ (the vector difference between $\boldsymbol{N}$ and $\boldsymbol{B}$ being $\boldsymbol{b}$ ). This geometry is shown in Fig. A1. Described here is a method by which $\boldsymbol{b}$ will be determined by future observations.

The nominal phase path difference, calculated using the nominal (assumed horizontal) baseline $N$ is:

$P_{N}=N \cos \theta=N . s$.

Similarly the real phase path difference is:

$P=\boldsymbol{B} . \boldsymbol{s}=(\boldsymbol{N}+\boldsymbol{b}) . s=\boldsymbol{N} . \boldsymbol{s}+\boldsymbol{b} . \boldsymbol{s}=P_{N}+\boldsymbol{b} . \boldsymbol{s}$,

where $s$ is a unit vector pointing towards the satellite. The rate of change of the observed phase is therefore:

$\frac{d \phi}{d t}=\left(\frac{2 \pi}{\lambda}\right) \frac{d P}{d t}=\left(\frac{2 \pi}{\lambda}\right) \frac{d P_{N}}{d t}+\left(\frac{2 \pi}{\lambda}\right) \frac{d(\boldsymbol{b} . \boldsymbol{s})}{d t}$.

The first term on the right hand side of Eq. (A3) is the rate of change of phase predicted using the nominal baseline and the second term is the "drift" correction $\left(\delta_{0}\right)$ required because the true baseline differs from the nominal one.

$$
\begin{aligned}
\delta_{0} & =\left(\frac{2 \pi}{\lambda}\right) \frac{d(\boldsymbol{b} . \boldsymbol{s})}{d t} \\
& =\left(\frac{2 \pi}{\lambda}\right)\left(b_{x} \frac{d s_{x}}{d t}+b_{y} \frac{d s_{y}}{d t}+b_{z} \frac{d s_{z}}{d t}\right)
\end{aligned}
$$




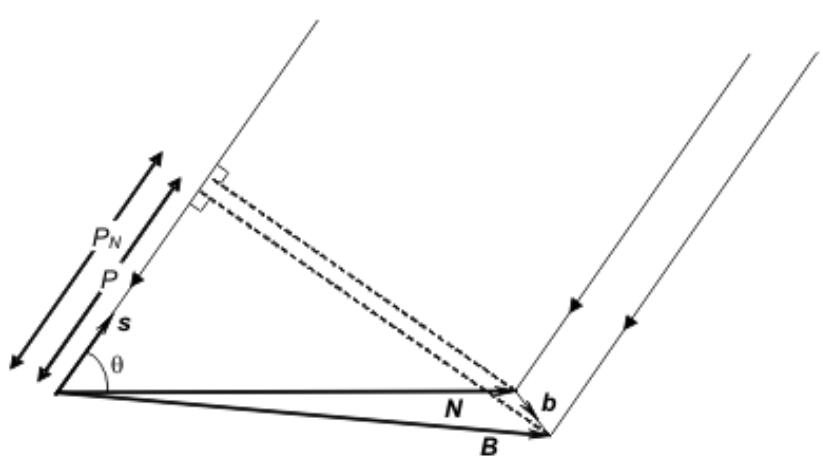

Fig. A1. Geometry of observations. $\boldsymbol{N}$ is the nominal (horizontal) baseline between the foci of the 42-m and 32-m antennae, $\boldsymbol{B}$ is the true baseline and $\boldsymbol{b}$ is the vector difference between the two. $\boldsymbol{s}$ is a unit vector pointing at the satellite. The true phase path difference is $P$, but that calculated assuming the nominal baseline is $P_{N}$.

where the three components of the unit vector $s$ obey $s_{x}^{2}+s_{y}^{2}+s_{z}^{2}=1$, so:

$s_{x} \frac{d s_{x}}{d t}+s_{y} \frac{d s_{y}}{d t}+s_{z} \frac{d s_{z}}{d t}=0$.

From Eqns. (A5) and (A4):

$\delta_{0}=\left(\frac{2 \pi}{\lambda}\right)\left(b_{x}-b_{z} \frac{s_{x}}{s_{z}}\right) \frac{d s_{x}}{d t}+\left(b_{y}-b_{z} \frac{s_{y}}{s_{z}}\right) \frac{d s_{y}}{d t}$.

Let us define the z-direction of the coordinate system to be in the field aligned direction and because all measurements are taken with the satellite within about $5^{\circ}$ of this direction $s_{z} \approx 1$ and $s_{x} \approx s_{y} \approx 0$. Thus we can approximate Eq. (A6) to:

$\delta_{0}=\left(\frac{2 \pi}{\lambda}\right)\left(b_{x} \frac{d s_{x}}{d t}+b_{y} \frac{d s_{y}}{d t}\right)$.

Thus to calculate $\delta_{0}$ we require knowledge of $b_{x}$ and $b_{y}$ and the satellite pass orientation and speed. If we select satellite passes that move through the centre of the beam, we can define the $\mathrm{x}$-direction to be that of the satellite pass, so $\frac{d s_{y}}{d t}=0$, and Eq. (A7) reduces to:

$b_{x}=\frac{\left(\lambda \delta_{0}\right)}{2 \pi\left(\frac{d s_{x}}{d t}\right)}$

from which the component of the correction vector $\boldsymbol{b}$, in the direction of satellite motion, can be calculated from the best fit drift $\delta_{0}$, given that $\frac{d s_{x}}{d t}$ is known. Using several passes of different orientations, $\boldsymbol{b}$ will be estimated.

To assess the accuracy of using this method, the fractional error $\varepsilon_{x}$ in the component $b_{x}$ is:

$\varepsilon_{x}=\left(\varepsilon_{\delta}^{2}+\varepsilon_{s}^{2}\right)^{\frac{1}{2}}$ where $\varepsilon_{\delta}$ is the fractional error in the determination of $\delta_{0}$ and $\varepsilon_{s}$ is the fractional error in $\frac{d s_{x}}{d t}$. If the (small) angle between the z-axis and $s$ is $\theta, s_{x}=\sin \theta$ and thus:

$\frac{d s_{x}}{d t}=\cos \theta \frac{d \theta}{d t} \approx \frac{d \theta}{d t}$.

Thus the fractional error in $\frac{d s_{x}}{d t}$ is the same as that in $\frac{d \theta}{d t}$. Using the example presented in this paper, the satellite moves across the Odin field of view through an angle $\eta$ of $12.35^{\circ}$, and the uncertainty in this angle $\varepsilon_{\eta}$ is one camera pixel width $\left( \pm 0.02^{\circ}\right)$. It moves across this angle in $15.96 \mathrm{~s}$ at an average angular speed of $\frac{d \eta}{d t}=0.774 \pm 0.001 \mathrm{deg} \mathrm{s}^{-1}$. Thus the fractional error in the angular speed of the satellite is $0.16 \%$. The angle $\eta$ in this case is not quite the same as $\theta$ because the satellite did not pass through the centre of the beam. Nevertheless, $\eta$ and $\theta$ are sufficiently similar that we can use the fractional uncertainty in $\eta$ as a typical of that in $\theta$ also (in fact because $\varepsilon_{\delta} \gg \varepsilon_{s}$, the difference between $\eta$ and $\theta$ is of no significance in this context). Hence we adopt $\varepsilon_{s}=0.0016$.

The error $\varepsilon_{\delta}$ can be estimated from Fig. 7, which shows that best-fit drift $\delta_{0}=0.625 \mathrm{rad} \mathrm{s}^{-1}$ with an uncertainty at the 1 sigma level (84\%) of between 0.46 and 0.82 , giving an average uncertainty of \pm 0.18 , i.e. a fractional uncertainty of 0.29 . From Eq. (A9), $\varepsilon_{s}$ is clearly negligible and $\varepsilon_{x}=\varepsilon_{\delta}=0.29$.

To estimate the absolute magnitude of this uncertainty, we note that Eq. (A8) gives $b_{x}$ of $4.42 \mathrm{~m}$ (although note that this satellite pass does not go through the centre of the beam and so Eq. (A8) is only approximately valid in this case), and thus a fractional error of $\varepsilon_{x}=0.29$ corresponds to an uncertainty of $1.27 \mathrm{~m}=2.12 \lambda$.

If the measurement is repeated for another pass in the same direction, the estimate and its uncertainty would be independent of the first measurement. Thus, with a set of, for example, $n=16$ such passes, the uncertainty in $b_{x}$ could be reduced by $n^{\frac{1}{2}}$ to $0.53 \lambda$.

Acknowledgements. With thanks to the January 2003 Interferometry Campaign Team from the University of Troms $\varnothing$. EISCAT is an International Association supported by Finland (SA), France (CNRS), Germany (MPG), Japan (NIPR), Norway (NFR), Sweden (VR) and the United Kingdom (PPARC).

Topical Editor M. Pinnock thanks two referees for their help in evaluating this paper.

\section{References}

Blixt, E. M., Grydeland, T., Ivchenko, N., Hagfors, T., La Hoz, C., Lanchester, B. S., Løvhaug, U. P., and Trondsen, T. S.: Dynamic rayed aurora and enhanced ion-acoustic radar echoes, Ann. Geophys., 23, 3-11, 2005, http://www.ann-geophys.net/23/3/2005/. 
Buchert, S. C., van Eyken, A. P., Ogawa, T., and Watanabe, S.: Naturally enhanced ion-acoustic lines seen with the EISCAT Svalbard Radar, Adv. Space Res., 23, 1699-1704, doi: 10.1016/S0273-1177(99)00382-8, 1999.

Cabrit, B., Opgenoorth, H., and Kofman, W.: Comparison between EISCAT UHF and VHF backscattering cross section, J. Geophys. Res., 101, 2369-2376, doi:10.1029/95JA02175, 1996.

Chen, J.-S., Röttger, J., and Chu, Y.-H.: System phase calibration of VHF spaced antennas using the echoes of aircraft and incorporating the frequency domain interferometry technique, Radio Sci., 37, 1080, doi:10.1029/2002RS002604, 2002.

Collis, P. N., Häggström, I., Kaila, K., and Rietveld, M. T.: EISCAT radar observations of enhanced incoherent scatter spectra; their relation to red aurora and field-aligned currents, Geophys. Res. Lett., 18, 1031-1034, 1991.

Foster, J. C., del Pozo, C., Groves, K., and St. Maurice, J.-P.: Radar observations of the onset of current driven instabilities in the topside ionosphere, Geophys. Res. Lett., 15, 160-163, 1988.

Grydeland, T., La Hoz, C., Hagfors, T., Blixt, E. M., Saito, S., Strømme, A., and Brekke, A.: Interferometric observations of filamentary structures associated with plasma instability in the auroral ionosphere, Geophys. Res. Lett., 30, 1338, doi:10.1029/ 2002GL016362, 2003.

Grydeland, T., Blixt, E. M., Løvhaug, U. P., Hagfors, T., La Hoz, C., and Trondsen, T. S.: Interferometric radar observations of filamented structures due to plasma instabilities and their relation to dynamic auroral rays, Ann. Geophys., 22, 1115-1132, 2004, http://www.ann-geophys.net/22/1115/2004/.
Holt, J. M., Erickson, P. J., Gorczyca, A. M., and Grydeland, T.: MIDAS-W: a workstation-based incoherent scatter radar data acquisition system, Ann. Geophys., 18, 1231-1241, 2000, http://www.ann-geophys.net/18/1231/2000/.

Lanchester, B. S., Rees, M. H., Robertson, S., Galand, D. L. M., Mendillo, M., Baumgardner, J., Furniss, I., and Aylward, A. D.: Proton and electron precipitation over Svalbard -first results from a new Imaging Spectrograph (HiTIES), in: Proc. of Atmos. Studies by Optical Methods, SGO Pubs 92, 33-36, 2003.

Rietveld, M. T., Collis, P. N., and St.-Maurice, J.-P.: Naturally Enhanced Ion Acoustic Waves in the Auroral Ionosphere Observed With the EISCAT 933-MHz Radar, J. Geophys. Res., 96, 19291$19305,1991$.

Sedgemore-Schulthess, K. J. F. and St.-Maurice, J.-P.: Naturally enhanced ion-acoustic spectra and their interpretation, Surv. Geophys., 22, 55-92, doi:10.1023/A:1010691026863, 2001.

Sedgemore-Schulthess, K. J. F., Lockwood, M., Trondsen, T. S., Lanchester, B. S., Rees, M. H., Lorentzen, D. A., and Moen, J.: Coherent EISCAT Svalbard Radar spectra from the dayside cusp/cleft and their implication for transient field-aligned currents, J. Geophys. Res., 104, 24 613-24 624, doi:10.1029/ 1999JA900276, 1999.

Sigernes, F., Svenøe, T., and Deehr, C. S.: The Auroral Station in Adventdalen, Svalbard, Chinese J. Pol. Sci., 13, 67-74, 2002.

Tereshchenko, E. D., Khudukon, B. Z., Rietveld, M. T., and Brekke, A.: Spatial structure of auroral day-time ionospheric electron density irregularities generated by a powerful HF-wave, Ann. Geophys., 16, 812-820, 1998, http://www.ann-geophys.net/16/812/1998/. 